PROCEEDINGS OF THE

AMERICAN MATHEMATICAL SOCIETY

Volume 137, Number 4, April 2009, Pages 1519-152

S 0002-9939(08)09721-9

Article electronically published on November 20, 2008

\title{
RATIONAL HOMOTOPY OF GAUGE GROUPS
}

\author{
YVES FÉLIX AND JOHN OPREA
}

(Communicated by Paul Goerss)

\begin{abstract}
In this brief paper, we observe that basic results from rational homotopy theory provide formulas for the rational homotopy groups of gauge groups of principal bundles $K \rightarrow P \rightarrow B$ in terms of the rational homotopy groups of $K$ and cohomology groups of $B$ alone.
\end{abstract}

\section{INTRODUCTION}

Let $K \rightarrow P \stackrel{\xi}{\rightarrow} B$ be a continuous principal $K$-bundle, where $K$ is a compact connected Lie group. Denote by $G(\xi)$ the gauge group of $\xi$ : that is, the set of all $K$ equivariant self-homeomorphisms of $P$ over $B$. Also, denote by $G_{1}(\xi)$ the subgroup of $G(\xi)$ consisting of the self-homeomorphisms that preserve the basepoint of $P$. (It is common in the subject to take for $G_{1}(\xi)$ the self-homeomorphisms that fix a given fibre $K$, but because $G(\xi)$ consists of equivariant maps, this is equivalent to our definition above.) The topology of gauge groups has been considered by many authors; see, for instance, [5, 2] or 11. Indeed, the study of the homotopy theory of gauge groups (under a different name) goes back to [5].

Now, there is an obvious homeomorphism of groups $G_{1}(\xi) \cong \operatorname{Map}_{*}(P, K)_{K}$, where the subscript $K$ on the mapping space denotes the space of equivariant maps with respect to the free (principal) action of $K$ on $P$ and the conjugation action of $K$ on $K$. In [16], S. Terzić shows that when $B$ is a closed simply connected 4-manifold, there is a formula for the ranks of the homotopy groups $\pi_{j}(G(\xi))$ and $\pi_{j}\left(G_{1}(\xi)\right)$ in terms of the ranks of the homotopy of $K$ and homology of $B$ alone (see Corollary 3.3).

Also, when $K$ is abelian, we clearly have $G_{1}(\xi)=\operatorname{Map}_{*}(P, K)_{K}=\operatorname{Map}_{*}(B, K)$. For $\operatorname{dim}(B) \leq 4$, it was shown in 12 that even when $K$ is non-abelian, there is a weak equivalence between $G_{1}(\xi)$ and $\operatorname{Map}_{*}(B, K)$. In certain cases, this result can be extended beyond these cases (see Corollary 2.2). Indeed, more recently, in [18, 19], C. Wockel shows that for principal bundles $K \rightarrow P \stackrel{\xi}{\rightarrow} S^{m}$, there is an identification of homotopy types $\operatorname{Map}_{*}(P, K)_{K} \simeq \operatorname{Map}_{*}\left(S^{m}, K\right)$ (and a consequent isomorphism $\pi_{q}\left(G_{1}(\xi)\right)=\pi_{q+m}(K)$ over $\left.\mathbb{Z}\right)$.

The purpose of this short paper is simply to observe that the philosophy of the preceding paragraph, in the framework of rational homotopy theory, allows the derivation of a general formula (see Theorem 3.1) for the rational homotopy

Received by the editors July 1, 2008, and, in revised form, August 4, 2008.

2000 Mathematics Subject Classification. Primary 55P99; Secondary 57R91, 57S05.

Key words and phrases. Gauge group, mapping space, principal bundle.

(C)2008 American Mathematical Society 
groups of the gauge groups $G(\xi)$ and $G_{1}(\xi)$ which, in particular, recovers Wockel's isomorphism (over the rationals) when the base is a sphere and specializes to the formula of Terzić when the base is a 4-manifold. Because these results are of interest to non-topologists, we have tried to include as many details as possible within the confines of a desire for conciseness.

\section{Homotopy type of GAuge Groups}

One basic result of the theory of gauge groups says the following.

Theorem 2.1. For a principal bundle $K \rightarrow P \stackrel{\xi}{\rightarrow} B$ with classifying map $f: B \rightarrow$ $B_{K}$,

$$
G(\xi)=\operatorname{Map}(P, K)_{K} \simeq \Omega \operatorname{Map}\left(B, B_{K} ; f\right)
$$

and

$$
G_{1}(\xi)=\operatorname{Map}_{*}(P, K)_{K} \simeq \operatorname{SMap}_{*}\left(B, B_{K} ; f\right) .
$$

This theorem may be proved in several ways. For instance, it was shown in [5. Theorems 5.2 and 5.6, Proposition 4.3] that $G(\xi)$ and $G_{1}(\xi)$ are the fibres in fibrations with the mapping $\operatorname{spaces} \operatorname{Map}\left(B, B_{K} ; f\right)$ and $\operatorname{Map}_{*}\left(B, B_{K} ; f\right)$, respectively, as base spaces and with (essentially) contractible total spaces. Also see [2, Theorem 3.3 and Corollary 5.7] and [11, Chapter 2].

In general, if $W$ has the homotopy type of an $H$-space (for instance, a topological group or a loop space), then all the components of $\operatorname{Map}(Z, W)$ have the same homotopy type, because multiplication with $f$ provides an equivalence $\operatorname{Map}(Z, W ; *) \cong$ $\operatorname{Map}(Z, W ; f)$. Furthermore, if $Z=\Sigma X$ is a suspension (or, more generally, if $Z$ is an associative co-H-space), then all components of $\operatorname{Map}_{*}(Z, W)=\operatorname{Map}_{*}(\Sigma X, W) \simeq$ $\operatorname{Map}_{*}(X, \Omega W ; f)$. Therefore, under these types of conditions, we have equivalences $\operatorname{Map}_{*}(Z, W ; f) \simeq \operatorname{Map}_{*}(Z, W ; *)$. Furthermore, we also have the general equality ${\Omega \operatorname{Map}_{*}}(Z, W ; f) \simeq \operatorname{SMap}_{*}(Z, W ; *)=\operatorname{Map}_{*}(Z, \Omega W)$. Thus, using the fact that $\Omega B_{K} \simeq K$, we obtain

Corollary 2.2 ([11, Theorem 2.2.4]). If all the components of $\operatorname{Map}_{*}\left(B, B_{K}\right)$ have the same homotopy type, then

$$
G_{1}(\xi) \simeq \operatorname{Map}_{*}(B, K)
$$

The hypothesis of Corollary 2.2 is not always satisfied. For instance, in 8 ] A. Kono constructs principal $S U(2)$-fibrations $\xi$ and $\xi^{\prime}$ over $S^{4}$ with $G(\xi) \neq G\left(\xi^{\prime}\right)$. In this case, all the components of $\operatorname{Map}\left(B, B_{K}\right)$ do not have the same homotopy type.

On the other hand, over the rational numbers we can generalize Corollary 2.2 to obtain

Theorem 2.3. When $B$ has the homotopy type of a connected finite $C W$ complex, there are rational homotopy equivalences

$$
G(\xi) \simeq_{\mathbb{Q}} \operatorname{Map}(B, K) \quad \text { and } \quad G_{1}(\xi) \simeq_{\mathbb{Q}} \operatorname{Map}_{*}(B, K) .
$$

Proof. The mapping space $\operatorname{Map}\left(B, B_{K} ; f\right)$ is a nilpotent space whose rationalization is the space $\operatorname{Map}\left(B,\left(B_{K}\right)_{\mathbb{Q}} ; f_{\mathbb{Q}}\right)$ (see [6, Theorems II.2.5 and II.3.11] and [7] for more details on free mapping spaces). Moreover, we know that the rational 
cohomology of $B_{K}$ is a polynomial algebra (see [4, Theorem 1.81 and Example 2.42] for example). More specifically, the cohomology of a compact connected Lie group is an exterior algebra $H^{*}(K ; \mathbb{Q})=\wedge\left(u_{1}, \ldots, u_{r}\right)$ with $u_{i} \in H^{2 n_{i}-1}(K ; \mathbb{Q})$, and the Serre spectral sequence for the universal bundle $K \rightarrow E_{K} \rightarrow B_{K}$ leads to $H^{*}\left(B_{K} ; \mathbb{Q}\right)=\mathbb{Q}\left[v_{1}, \ldots, v_{r}\right]$, a polynomial algebra with $v_{i} \in H^{2 n_{i}}\left(B_{K} ; \mathbb{Q}\right)$. Since cohomology corresponds in general to homotopy classes of maps, we have $H^{2 n_{i}}\left(B_{K} ; \mathbb{Q}\right)=\left[B_{k}, K\left(\mathbb{Q}, 2 n_{i}\right)\right]$, for each $i=1, \ldots, r$. We then obtain a map

$$
B_{K} \rightarrow \prod_{i=1}^{r} K\left(\mathbb{Q}, 2 n_{i}\right)
$$

which clearly induces an isomorphism on rational cohomology (as well as homology) and is, therefore, a rational equivalence. Hence, the rationalization $\left(B_{K}\right)_{\mathbb{Q}}$ is an $H$-space (since a product of $K(\mathbb{Q}, j)$ 's clearly is), so $\operatorname{Map}\left(B,\left(B_{K}\right)_{\mathbb{Q}} ; f_{\mathbb{Q}}\right)$ has the homotopy type of $\operatorname{Map}\left(B,\left(B_{K}\right)_{\mathbb{Q}} ; *\right)$. Then

$$
G(\xi) \simeq \Omega \operatorname{Map}\left(B, B_{K} ; f\right) \simeq_{\mathbb{Q}} \Omega \operatorname{Map}\left(B,\left(B_{K}\right)_{\mathbb{Q}} ; *\right) \simeq \operatorname{Map}\left(B, K_{\mathbb{Q}}\right),
$$

since $\Omega B_{K} \simeq K$.

The exact same argument applies to the based mapping space $\operatorname{Map}_{*}\left(B, B_{K} ; f\right)$ and $G_{1}(\xi)$.

Remark 2.4. The same argument as in the proof applied to the cohomology algebra $H^{*}(K ; \mathbb{Q})=\wedge\left(u_{1}, \ldots, u_{r}\right)$ of a compact connected Lie group $K$ shows that, rationally, $K$ is also a product of Eilenberg-Mac Lane spaces: that is,

$$
K_{\mathbb{Q}} \simeq \prod_{i=1}^{r} K\left(\mathbb{Q}, 2 n_{i}-1\right)
$$

\section{RATional hOMOtopy of GAUGe GROUPS}

We now use Theorem 2.3 to compute rational homotopy groups of gauge groups for any finite connected base space $B$. Recall that $K \rightarrow P \stackrel{\xi}{\rightarrow} B$ is a continuous principal bundle with $K$ a compact connected Lie group.

Theorem 3.1. If $B$ has the homotopy type of a finite connected $C W$ complex, then for any $q \geq 1$, we have

$$
\pi_{q}(G(\xi)) \otimes \mathbb{Q} \cong \sum_{r \geq 0} H^{r}(B ; \mathbb{Q}) \otimes \pi_{r+q}(K)
$$

and

$$
\pi_{q}\left(G_{1}(\xi)\right) \otimes \mathbb{Q} \cong \sum_{r \geq 0} \widetilde{H}^{r}(B ; \mathbb{Q}) \otimes \pi_{r+q}(K),
$$

where $\widetilde{H}$ denotes reduced cohomology.

Remark 3.2. Note that since $H^{r}(B ; \mathbb{Q})$ is a rational vector space, there is no need to write $\pi_{r+q}(K) \otimes \mathbb{Q}$. Also, recall that for path-connected $X$, the term reduced cohomology means that $\widetilde{H}^{j}(X)=H^{j}(X)$ for $j \geq 1$ and $\widetilde{H}^{0}(X)=0$. Finally, there has been much work on the rational homotopy groups of mapping spaces. See, for example, [17, 10, 3, 1. Each of these papers uses the minimal model theory of Sullivan, but in the following proof, the fact that $K$ is very simple over $\mathbb{Q}$ allows us to take a more elementary approach. 
Proof. By Theorem 2.3 we have the rational equivalences $G(\xi) \simeq_{\mathbb{Q}} \operatorname{Map}(B, K)$ and $G_{1}(\xi) \simeq_{\mathbb{Q}} \operatorname{Map}_{*}(B, K)$. Consider $\pi_{q}\left(G_{1}(\xi)\right) \otimes \mathbb{Q}=\pi_{q}\left(\operatorname{Map}_{*}(B, K)\right) \otimes \mathbb{Q}$. Because it is true in general that $\pi_{q}\left(\operatorname{Map}_{*}(X, Y)\right)=\left[\Sigma^{q} X, Y\right]$, and by the universal property of localization we have

$$
\begin{aligned}
\pi_{q}\left(\operatorname{Map}_{*}(B, K)\right) \otimes \mathbb{Q} & =\left[\Sigma^{q} B, K\right]_{\mathbb{Q}}=\left[\Sigma^{q} B, K_{\mathbb{Q}}\right] \\
& =\left[\Sigma^{q} B, \prod_{i=1}^{r} K\left(\mathbb{Q}, 2 n_{i}-1\right)\right] \quad \text { by Remark } 2.4 \\
& =\prod_{i=1}^{r}\left[\Sigma^{q} B, K\left(\mathbb{Q}, 2 n_{i}-1\right)\right] \\
& =\bigoplus_{i=1}^{r} H^{2 n_{i}-1-q}(B ; \mathbb{Q}) \\
& =\bigoplus_{r \geq 0} \widetilde{H}^{r}(B ; \mathbb{Q}) \otimes \pi_{r+q}(K),
\end{aligned}
$$

where, in the last line, we have replaced $2 n_{i}-1$ by $r+q$ and recognized that the only non-zero terms occur in degrees $j$ where $\pi_{j}(K) \otimes \mathbb{Q} \neq 0$.

Now, for $\mathcal{H}$ an $H$-space, we always have the following relationship between free and based mapping spaces (see [9, Proposition 4.9] or [7] for instance):

$$
\operatorname{Map}(X, \mathcal{H} ; *) \simeq \mathcal{H} \times \operatorname{Map}_{*}(X, \mathcal{H} ; *) .
$$

Thus, since all components of $\operatorname{Map}\left(B,\left(B_{K}\right)_{\mathbb{Q}}\right)$ have the same homotopy type, we can apply this result to see that

$$
\pi_{q}(G(\xi)) \otimes \mathbb{Q}=\pi_{q}\left(\operatorname{Map}\left(B, K_{\mathbb{Q}} ; *\right)\right)=\pi_{q}(K) \otimes \mathbb{Q} \oplus \pi_{q}\left(\operatorname{Map}_{*}\left(B, K_{\mathbb{Q}} ; *\right)\right),
$$

and the formula for $\pi_{q}(G(\xi)) \otimes \mathbb{Q}$ follows.

Of course, if $B=S^{m}$, then we recover Wockel's result over $\mathbb{Q}, \pi_{q}\left(G_{1}(\xi)\right) \otimes \mathbb{Q}=$ $\pi_{q+m}(K) \otimes \mathbb{Q}$. Moreover, if the base $B$ of the principal bundle is a closed simply connected 4-manifold, then $H^{1}(B ; \mathbb{Q})=0=H^{3}(B ; \mathbb{Q})$ and $H^{4}(B ; \mathbb{Q})=\mathbb{Q}$. Hence, from the general formulas above, we obtain

Corollary 3.3 (Terzić's Formula [16, Propositions 1 and 2]). If $K \rightarrow P \rightarrow B$ is a principal bundle (as above) with $B$ a closed simply connected 4-manifold with second Betti number $b_{2}(B)$, then

$$
\operatorname{rank}\left(\pi_{q}(G(\xi))\right)=b_{2}(B) \cdot \operatorname{rank}\left(\pi_{q+2}(K)\right)+\operatorname{rank}\left(\pi_{q+4}(K)\right)+\operatorname{rank}\left(\pi_{q}(K)\right)
$$

and

$$
\operatorname{rank}\left(\pi_{q}\left(G_{1}(\xi)\right)\right)=b_{2}(B) \cdot \operatorname{rank}\left(\pi_{q+2}(K)\right)+\operatorname{rank}\left(\pi_{q+4}(K)\right) .
$$

Note that because the formula only involves ranks of homotopy groups, it is in fact a result about rational homotopy groups. Also, since the non-zero rational homology of $B$ occurs only in even-degrees and the non-zero rational homotopy of $K$ occurs only in odd degrees, all even-degree rational homotopy of the gauge groups vanishes. For the same reasons, this will also be true whenever $B$ has $H^{\text {odd }}(B ; \mathbb{Q})=0$. In particular, we have 
Example 3.4. If $K \rightarrow P \stackrel{\xi}{\rightarrow} \mathbb{C} P^{m}$ is a principal bundle, then $\pi_{q}(G(\xi)) \otimes \mathbb{Q}=0=$ $\pi_{q}\left(G_{1}(\xi)\right) \otimes \mathbb{Q}$ for $q$ even and, for $q$ odd,

$$
\pi_{q}(G(\xi)) \otimes \mathbb{Q}=\bigoplus_{i=0}^{m} \pi_{q+2 i}(K) \otimes \mathbb{Q} \quad \text { and } \quad \pi_{q}\left(G_{1}(\xi)\right) \otimes \mathbb{Q}=\bigoplus_{i=1}^{m} \pi_{q+2 i}(K) \otimes \mathbb{Q}
$$

These observations can be put in a wider context. A space $B$ is said to be rationally elliptic if its rational homotopy and rational homology are both finite dimensional. For instance, spheres and homogeneous spaces are rationally elliptic. If a rationally elliptic $B$ also has positive Euler characteristic, $\chi(B)>0$, then it is known that $H^{\text {odd }}(B ; \mathbb{Q})=0$ (see [4, Theorem 2.75] for instance). Therefore, by the discussion above,

$$
\pi_{\text {even }}(G(\xi)) \otimes \mathbb{Q}=0=\pi_{\text {even }}\left(G_{1}(\xi)\right) \otimes \mathbb{Q}
$$

for any principal bundle $K \rightarrow P \stackrel{\xi}{\rightarrow} B$. The connection to geometry arises from two sources (see [4, Section 6.4] for a fuller discussion). First, there is the conjecture of Raoul Bott that compact manifolds of positive sectional curvature are rationally elliptic. Second, there is the conjecture of Heinz Hopf that even-dimensional compact manifolds of positive sectional curvature have positive Euler characteristics. If both these conjectures are true, then by what we have said above, the evendegree rational homotopy groups of gauge groups vanish. This elicits the following question:

Question 3.5. Let $K \rightarrow P \stackrel{\xi}{\rightarrow} B$ be a principal bundle. If $B^{2 m}$ is a compact manifold with positive sectional curvature (in some metric), then is it true that

$$
\pi_{\text {even }}(G(\xi)) \otimes \mathbb{Q}=0=\pi_{\text {even }}\left(G_{1}(\xi)\right) \otimes \mathbb{Q} ?
$$

\section{The gauge group of the universal Bundle}

Because it classifies all principal $K$-bundles, the most important principal bundle is the universal bundle $\xi_{u}: K \rightarrow E_{K} \rightarrow B_{K}$. Thus, its gauge group is of interest. Unfortunately, in the proof of Theorem 3.1, we needed the base space of the bundle to be a finite complex in order to be able to localize mapping spaces. Of course, this is not the case for $B_{K}$. Nevertheless, we can still compute the rational homotopy groups of $G\left(\xi_{u}\right)$ by making use of the more algebraic framework of rational homotopy theory (see 4 for instance) and, in particular, a theorem of Smith 13 .

Let $\operatorname{aut}_{1}(X)=\operatorname{Map}\left(X, X ; 1_{X}\right)$, the monoid of self-homotopy equivalences of $X$ homotopic to the identity $1_{X}$. There is a classifying space $\operatorname{Baut}_{1}(X)$ with the usual property that $\Omega \operatorname{Baut}_{1}(X)=\operatorname{aut}_{1}(X)$. There is a general way to study the rational homotopy type of $\operatorname{Baut}_{1}(X)$ from the viewpoint of commutative differential graded algebras and differential graded Lie algebras (see, for instance, [14, 15]). This viewpoint equates the rational homotopy groups with the homology of the complex of degree-lowering derivations on the minimal model of $X$. We will not go into details about models since the following special case is all that we need.

Theorem 4.1 ([13, Theorem 2 and Corollary 2]). If $X$ is a rational $H$-space of finite type, then

$$
\pi_{q}\left(\operatorname{aut}_{1}(X)\right) \otimes \mathbb{Q}=\pi_{q}\left(\Omega \operatorname{Baut}_{1}(X)\right) \otimes \mathbb{Q}=\operatorname{Der}^{q}\left(H^{*}(X ; \mathbb{Q})\right),
$$

where $\operatorname{Der}^{q}\left(H^{*}(X ; \mathbb{Q})\right)$ is the vector space of derivations on the cohomology algebra which lower degree by $q$. 
Recall that $X$ is a rational $H$-space if its $\mathbb{Q}$-localization is an $H$-space. In fact, we have already used the fact that $B_{K}$ is a rational $H$-space of finite type in the proof of Theorem 2.3. so Theorem 4.1 applies to $B_{K}$. Indeed, as we described in the proof of Theorem $2.3, H^{*}\left(B_{K} ; \mathbb{Q}\right)=\mathbb{Q}\left[v_{1}, \ldots, v_{r}\right]$, a polynomial algebra with $v_{i} \in H^{2 n_{i}}\left(B_{K} ; \mathbb{Q}\right)$; so Hopf's classification says that $B_{K} \simeq_{\mathbb{Q}} \prod_{i} K\left(\mathbb{Q}, 2 n_{i}\right)$, with the $v_{i}$ 's corresponding to a basis for the rational homotopy groups. Therefore $B_{K}$ is an $H$-space after rationalization.

Now, by Theorem 2.1, the gauge group of the universal bundle is given by $G\left(\xi_{u}\right)=\Omega \operatorname{Map}\left(B_{K}, B_{K} ; 1\right)=\Omega\left(\right.$ aut $\left._{1} B_{K}\right)$. By Theorem 4.1, we can then compute the gauge group from the derivations of cohomology $\operatorname{Der}^{*}\left(H^{*}\left(B_{K} ; \mathbb{Q}\right)\right)=$ $\operatorname{Der}^{*}\left(\mathbb{Q}\left[u_{\alpha}\right]\right)$. The derivations of this algebra are particularly easy to understand. In particular, a basis for the derivations which lower degree by $q+1$ consists of those derivations that are non-zero on a single generator $u_{t}$ (in degree $t$, say) and have image any element in degree $t-q-1$. Since the $u_{\alpha}$ generate $\pi_{\alpha}\left(B_{K}\right)$ and $\mathbb{Q}\left[u_{\alpha}\right]=H^{*}\left(B_{K} ; \mathbb{Q}\right)$, we can make the identification

$$
\operatorname{Der}^{q+1}\left(\mathbb{Q}\left[u_{\alpha}\right]\right)=\bigoplus_{t \geq 0} H^{t-q-1}\left(B_{K} ; \mathbb{Q}\right) \otimes \pi_{t}\left(B_{K}\right) .
$$

We then obtain the same formula as in Theorem 3.1, but now for the universal bundle having infinite-dimensional base $B_{K}$.

\section{Theorem 4.2.}

$$
\pi_{q}\left(G\left(\xi_{u}\right)\right) \otimes \mathbb{Q}=\bigoplus_{r \geq 0} H^{r}\left(B_{K} ; \mathbb{Q}\right) \otimes \pi_{q+r}(K)
$$

Proof.

$$
\begin{aligned}
\pi_{q}\left(G\left(\xi_{u}\right)\right) \otimes \mathbb{Q} & =\pi_{q}\left(\Omega\left(\operatorname{aut}_{1} B_{K}, 1\right)\right) \otimes \mathbb{Q} \\
& =\pi_{q+1}\left(\operatorname{aut}_{1} B_{K}, 1\right) \otimes \mathbb{Q} \\
& =\operatorname{Der}^{q+1}\left(\mathbb{Q}\left[u_{\alpha}\right]\right) \\
& =\bigoplus_{t} H^{t-q-1}\left(B_{K} ; \mathbb{Q}\right) \otimes \pi_{t}\left(B_{K}\right) \\
& =\bigoplus_{r} H^{r}\left(B_{K} ; \mathbb{Q}\right) \otimes \pi_{q+r}(K),
\end{aligned}
$$

where we have used the general facts that $\pi_{j}(\Omega X)=\pi_{j+1}(X)$ and $\pi_{j}(K)=$ $\pi_{j+1}\left(B_{K}\right)$. This is, of course, the same result as in Theorem 3.1 .

The gauge group has many equivalent definitions (see [11, Chapter 2]). For the universal bundle, the most homotopically interesting one is

$$
G\left(\xi_{u}\right)=\operatorname{Map}\left(E_{K}, K\right)_{K}
$$

the mapping space of equivariant maps $E_{K} \rightarrow K$, where the action on $K$ is by conjugation. In homotopy theory, this is exactly the definition of the homotopy fixed set $K^{h K}$ of the conjugation action. In general, it is very difficult to obtain explicit information about $K^{h K}$. Here, as a byproduct, we find

Corollary 4.3. Let $K$ act on itself by conjugation. Then the rational homotopy groups of the homotopy fixed set are given by

$$
\pi_{q}\left(K^{h K}\right) \otimes \mathbb{Q}=\bigoplus_{r \geq 0} H^{r}\left(B_{K} ; \mathbb{Q}\right) \otimes \pi_{q+r}(K) .
$$


The homotopy fixed set $K^{h K}$ (and $G\left(\xi_{u}\right)$ ) may also be identified with the space of sections of the associated fibre bundle $K_{K} \stackrel{\text { def }}{=} E_{K} \times_{K} K \rightarrow B_{K}$, where again the action of $K$ on itself is by conjugation. Yet one more tantalizing connection arises from the following folklore identification. Because we cannot find a reference, we give a brief outline of the proof.

Lemma 4.4. The bundle $K_{K}=E_{K} \times_{K} K \rightarrow B_{K}$ is homotopy equivalent to the free loop space fibration $B_{K}^{S^{1}} \rightarrow B_{K}$.

Proof. We outline the proof in steps.

Step 1. First define an action $(K \times K) \times K \rightarrow K$ by $(g, h) \cdot k=g \cdot k \cdot h^{-1}$. Then it is straightforward to show that $\phi:\left(E_{K} \times E_{K}\right) / K \rightarrow K_{(K \times K)}, \phi([x, y])=[x, y, e]$, is a homeomorphism, where $K$ acts on $E_{K} \times E_{K}$ diagonally (on the right in both factors), $K_{(K \times K)}$ is the Borel construction for the action defined above and $e$ is the identity of $K$. An inverse is given by $\phi^{-1}([x, y, e])=[x, y]$.

Step 2. Define a map $\theta:\left(E_{K} \times E_{K}\right) / K \rightarrow B_{K}$ by composing $\phi$ with $K_{(K \times K)} \rightarrow$ $B_{(K \times K)} \simeq B_{K} \times B_{K} \rightarrow B_{K}$, where the last map is projection onto the first factor. The fibre is $F=\{[\bar{x}, \bar{y}] \mid[x]=[\bar{x}]\}$, where $[x]$ is fixed in $B_{K}$. Define maps $\beta: E_{K} \rightarrow$ $F$ and $\gamma: F \rightarrow E_{K}$ by $\beta(y)=[x, y]$ and $\gamma([\bar{x}, \bar{y}])=\bar{y} \cdot k$ where $k$ is the unique element of $K$ such that $\bar{x}=x \cdot k$. Notice that we are using the fact that $K$ acts freely on $E_{K}$. We then see that $F=E_{K}$ and, since $\theta$ is a fibration, $\left(E_{K} \times E_{K}\right) / K \simeq B_{K}$. Note that a homotopy inverse to $\theta$ is given by $\sigma: B_{K} \rightarrow\left(E_{K} \times E_{K}\right) / K, \sigma([x])=[x, x]$. Furthermore, note that the following triangle commutes (where $\Delta$ is the diagonal, $\Delta(z)=(z, z))$.

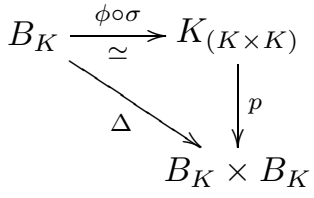

since $p \phi \sigma([x])=p([x, x, e])=[x, x]=([x],[x])$.

Step 3. Note that $K_{K}$ consists of elements $[x, k]$ with $[x, k]=[\bar{x}, \bar{k}]$ if and only if there is $h \in K$ such that $x \cdot h=\bar{x}$ and $h k h^{-1}=\bar{k}$. Define $\psi: K_{K} \rightarrow K_{(K \times K)}$ by $\psi([x, y]=[x, x, y]$. Then the following square is a pullback (and a homotopy pullback since $K_{(K \times K)} \rightarrow B_{K} \times B_{K}$ is a fibration).

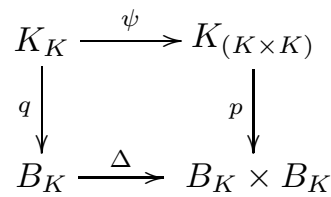

Since it is a homotopy pullback, we can replace $K_{(K \times K)} \rightarrow B_{K} \times B_{K}$ by the homotopy equivalent $\Delta: B_{K} \rightarrow B_{K} \times B_{K}$ as shown in Step 2. We therefore obtain $K_{K}$ as the homotopy pullback of $\Delta$ over itself. But this is well-known (see [4, Theorem 5.11]); specifically, we have the following homotopy commutative diagram where the left square is a homotopy pullback (and the vertical maps are the usual 
evaluations):

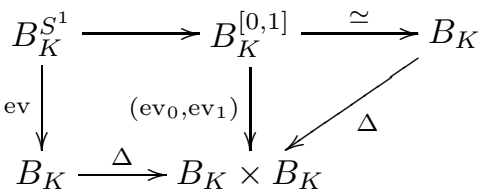

Thus, the free loop space $B_{K}^{S^{1}}$ also arises as the homotopy pullback of $\Delta$ over itself. Hence, $B_{K}^{S^{1}} \simeq K_{K}$.

Note that the free loop space has played and continues to play important roles in both geometry and homotopy theory (see 4] for example). Finally, we have the identification of the gauge group of the universal bundle with the space of sections of the free loop fibration on $B_{K}$,

$$
G\left(\xi_{u}\right)=\Gamma\left(B_{K}^{S^{1}} \rightarrow B_{K}\right) .
$$

By Theorem 4.2 we then know the rational homotopy groups of this intriguing space of sections.

\section{ACKNOWLEDGEMENTS}

We thank the referee for several helpful comments and suggestions.

\section{REFERENCES}

[1] J. Block and A. Lazarev, André-Quillen cohomology and rational homotopy of function spaces, Adv. Math. 193 (2005), no. 1, 18-39. MR2132759(2006a:55014)

[2] P. Booth, P. Heath, C. Morgan and R. Piccinini, H-spaces of self-equivalences of fibrations and bundles, Proc. London Math. Soc. 49 (1984), 111-127. MR743373 (85k:55013)

[3] U. Buijs and A. Murillo, Basic constructions in rational homotopy theory of function spaces, Ann. Inst. Fourier (Grenoble) 56 (2006), no. 3, 815-838. MR2244231 (2007h:55009)

[4] Y. Félix, J. Oprea and D. Tanré, Algebraic models in geometry, Oxford Graduate Texts in Mathematics 17, Oxford University Press, Oxford (2008). MR2403898

[5] D. Gottlieb, Applications of bundle map theory, Trans. Amer. Math. Soc. 171 (1972), 23-50. MR0309111 (46:8222)

[6] P. Hilton, G. Mislin and J. Roitberg, Localization of nilpotent groups and spaces, NorthHolland Math. Studies, No. 15. Notas de Matemática, No. 55. North-Holland Publishing Co., Amsterdam-Oxford; American Elsevier Publishing Co., Inc., New York (1975). MR0478146 $(57: 17635)$

[7] P. Hilton, G. Mislin, J. Roitberg and R. Steiner, On free maps and free homotopies into nilpotent spaces. Algebraic Topology (Proc. Conf., Univ. British Columbia, Vancouver, B.C., 1977), Lecture Notes in Math. 673, Springer, Berlin (1978), 202-218. MR517093 (80c:55007)

[8] A. Kono, A note on the homotopy type of certain gauge groups, Proc. Roy. Soc. Edinburgh 117 Sect. A (1991) 295-297. MR.1103296 (92b:55005)

[9] G. Lupton, C. Phillips, C. Schochet and S. Smith, Banach algebras and rational homotopy theory, Trans. Amer. Math. Soc. 361 (2009), 267-295.

[10] G. Lupton and S. Smith, Rationalized evaluation subgroups of a map. I. Sullivan models, derivations and G-sequences, J. Pure Appl. Algebra 209 (2007), no. 1, 159-171. MR2292124 (2008c:55017)

[11] R. Piccinini and M. Spreafico, Conjugacy classes in gauge groups, Queen's Papers in Pure and Applied Mathematics 111, Queen's University, Kingston, Ontario (1998). MR1687727 (2000f:55007)

[12] I. M. Singer, Some remarks on the Gribov ambiguity, Commun. Math. Physics 60 (1978), 7-12. MR.500248 (80d:53025)

[13] S. Smith, Rational type of classifying spaces for fibrations, Groups of homotopy selfequivalences and related topics (Gargnano, 1999), Contemp. Math. 274, Amer. Math. Soc., Providence, RI (2001), 299-307. MR.1817019 (2001m:55035) 
[14] D. Sullivan, Infinitesimal computations in topology, Inst. Hautes Étud. Sci. Publ. Math. 47 (1977), 269-331. MR0646078 (58:31119)

[15] D. Tanré, Homotopie rationelle: modèles de Chen, Quillen, Sullivan, Lecture Notes in Mathematics 1025, Springer-Verlag, Berlin (1983). MR764769 (86b:55010)

[16] S. Terzić, The rational topology of gauge groups and of spaces of connections, Compositio Math. 141 (2005), 262-270. MR2099779 (2005h:55011)

[17] M. Vigué-Poirrier, Sur l'homotopie rationnelle des espaces fonctionnels. Manuscripta Math. 56 (1986), 177-191. MR850369 (87h:55009)

[18] C. Wockel, The Samelson product and rational homotopy for gauge groups, Abh. Math. Sem. Univ. Hamburg 77 (2007), 219-228. MR2379340

[19] C. Wockel, Infinite dimensional Lie theory for gauge groups, Dissertation, Technische Universität Darmstadt (2006).

Institut de Mathématique Pure et Appliquée, Chemin du Cyclotron, 2, B-1348 Louvain-LA-Neuve, Belgium

E-mail address: Yves.Felix@uclouvain.be

Department of Mathematics, Cleveland State University, Cleveland, Ohio 44115

E-mail address: j.oprea@csuohio.edu 